\title{
Terahertz Imaging of ICs Using $K$-means Clustering for Scale-Invariant Feature Transform Feature and Fusion Model of Wavelet Transform
}

\author{
Xiao-Xiang Pu, ${ }^{1}$ Chih-Cheng Chen,,${ }^{2 *}$ Tie-Jun Li, ${ }^{3}$ Wei-Lung Mao, ${ }^{4 * *}$ and Zhen $\mathrm{Liu}^{3}$ \\ ${ }^{1}$ Chongqing Electric Power College, Chongqing 400053, China \\ ${ }^{2}$ Department of Automatic Control Engineering, Feng Chia University, Taichung 40724, Taiwan \\ ${ }^{3}$ School of Ocean Information Engineering, Jimei University, Fujian Province, Xiamen 361021, China \\ ${ }^{4}$ Department of Electrical Engineering, National Yunlin University of Science and Technology, \\ Yunlin 64002, Taiwan, R.O.C. \\ ${ }^{5}$ Department of Aeronautical Engineering, Chaoyang University of Technology, Taichung 413, Taiwan
}

(Received July 1, 2021; accepted November 22, 2021)

Keywords: terahertz spectral image, IC chip, SIFT feature, wavelet fusion

Terahertz spectral imaging is widely used in the failure analysis of ICs. However, owing to the limitations of current imaging hardware, the quality of terahertz images is not high enough to show the internal structure of chips and analyze chip failure. Thus, to improve the detection capability of chip failure in different packaging materials, we propose a terahertz imaging model based on scale-invariant feature transform (SIFT) feature extraction with the $K$-means clustering of images from multiple sources and the wavelet fusion method. The model creates a terahertz image data set from images drawn from multiple sources in the time and frequency domains. Then, the images drawn from the multiple sources are compared to select a representative image, and the SIFT features of the image are also extracted. The high-quality images obtained from multiple sources are searched and selected by $K$-means clustering. The images are reconstructed by wavelet image fusion. Experimental results on terahertz imaging in various packaging materials show that the model can quickly and effectively create high-quality images in the internal structure of ICs, which is essential for the nondestructive analysis of chip failures.

\section{Introduction}

IC chips are widely used in MEMS to achieve high performance in image-based detection, pressure sensing, acceleration, and so forth. However, the manufacturing of IC chips involves tens of operations and various devices, and this complex process may generate defects inside or on the surfaces of the chips. Thus, failure analysis is important in the production and use of semiconductor chips. Electrical function tests and the microscopic examination of fragments are commonly used methods in chip failure analysis. ${ }^{(1,2)}$ Recently, the development of terahertz technology has contributed to improving the technique of nondestructive analysis. Many studies have used terahertz spectral images for the failure analysis of IC chips. ${ }^{(3-5)}$ However, the optical

\footnotetext{
*Corresponding author: e-mail: chenccheng@fcu.edu.tw

** Corresponding author: e-mail: wlmao@yuntech.edu.tw https://oi.org/10.18494/SAM.2021.3521
} 
resolution of terahertz time-domain spectroscopy (THz-TDS) is not high enough to provide the terahertz image quality required for nondestructive tests. Therefore, improved image resolution, a key indicator in tests, is required for the accurate identification of the internal faults of IC chips. ${ }^{(6)}$

To develop a new method of terahertz imaging, we obtain terahertz images of IC chips in different packaging materials by various imaging methods and analyze them quantitatively to select the images with high resolution and quality. To acquire high-quality images for nondestructive analysis, a large number of terahertz images must be processed effectively and efficiently. Therefore, we propose a matching method for terahertz spectral images based on the combination of scale-invariant feature transform (SIFT) ${ }^{(7)}$ feature extraction and $K$-means clustering. ${ }^{(8)}$ The model processes terahertz images efficiently and generates high-quality images, enabling the internal faults of IC chips appearing at multiple angles and in different channels to be revealed. The clustering of similar images ensures the efficiency and effectiveness of fusion processing from the image source. Experimental results show that the new method provides terahertz images of high quality that are appropriate for nondestructive analysis and has better performance than the previous method of processing single terahertz images. ${ }^{(9-11)}$

\section{Methods}

\subsection{System model}

To select high-quality terahertz images, IC chips are scanned by a spectral imaging system. Then, the spectral data of each scanned pixel of an image in the time domain are obtained and processed by the windowed Fourier transform, which transforms the spectrum to the frequency domain. The data of the images are included in an image data set for nondestructive tests of IC chips. Next, to select a representative image from the data set that can best reflect the failure characteristics, the images are evaluated according to the terahertz spectrum and various evaluation parameters. Then, the SIFT feature- $K$-means extraction method is used to identify a group of similar images from the image data set. Finally, wavelet fusion is used to reconstruct an image of high quality. The overall process of the model is shown in Fig. 1.

\subsection{Multimodal imaging}

Terahertz spectral imaging is different from light intensity information imaging as it obtains the time-domain waveform and the electric field intensity of each pixel of a sample image in the entire terahertz band. The Fourier transform on the terahertz time-domain spectrum allows the frequency-domain spectrum of each scanned pixel to be acquired. Through the selection of different physical parameters in the time and frequency domains, a two-dimensional (2D) image of different spectral resolution is obtained. Terahertz spectral imaging is mainly divided into time- and frequency-domain methods. 


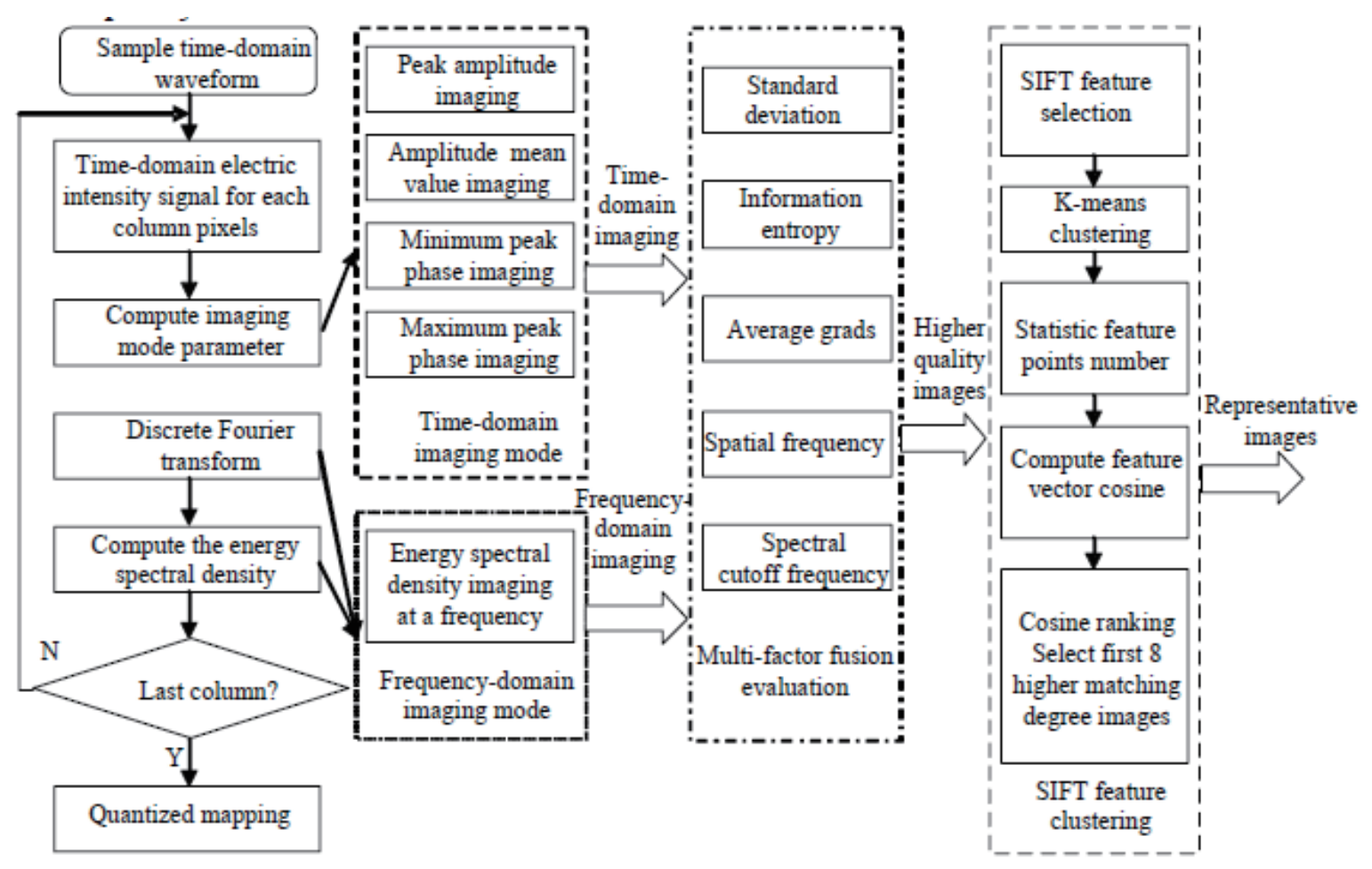

Fig. 1. Flowchart of selecting the representative image of an IC chip by terahertz imaging based on SIFT featureK-means clustering.

\subsubsection{Time-domain information}

The time-domain method mainly uses the physical parameters of a time-domain waveform. Amplitude and phase are two commonly used parameters. The amplitude of an image, which reflects the absorption strength of the image in the terahertz wave, refers to the peak and average value of the field strength in the time-domain waveform. The phase indicates the time corresponding to the minimum or maximum peak of the amplitude. Figure 2 shows the amplitude of terahertz imaging, in which $a$ is the maximum peak revealing the characteristics of image refraction and scattering, and $A$ is the time when the minimum peak occurs.

\subsubsection{Frequency-domain information}

Frequency-domain information is obtained from the physical parameters of frequencydomain spectra. These are amplitude, phase, energy, and absorption coefficient at a specific frequency and reflect the differences in the physical characteristics of images at different frequencies. Figure 3 shows the change in power spectral density with the frequency, in which B indicates the power spectral density.

Since the terahertz energy spectrum reflects the absorption of the terahertz wave by the image, we use the energy parameter of the energy density spectrum. The energy parameter is calculated by using Parseval's theorem, 


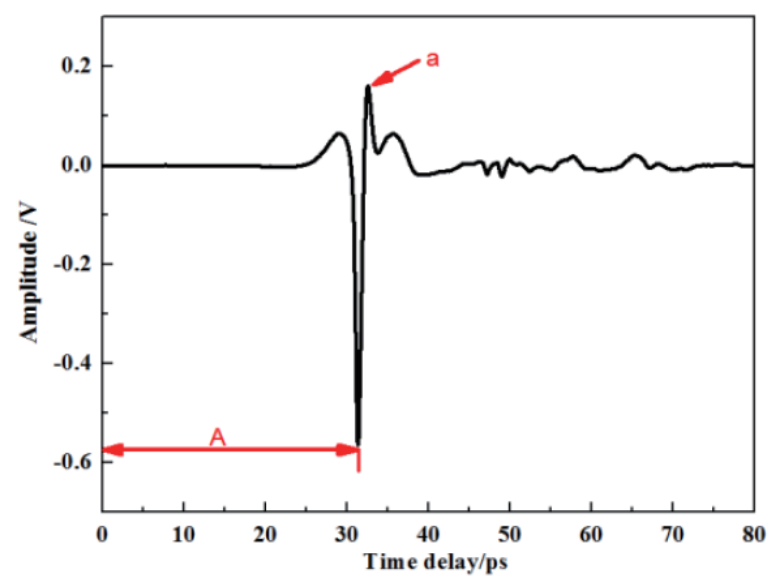

Fig. 2. (Color online) Information of terahertz imaging in the time domain.

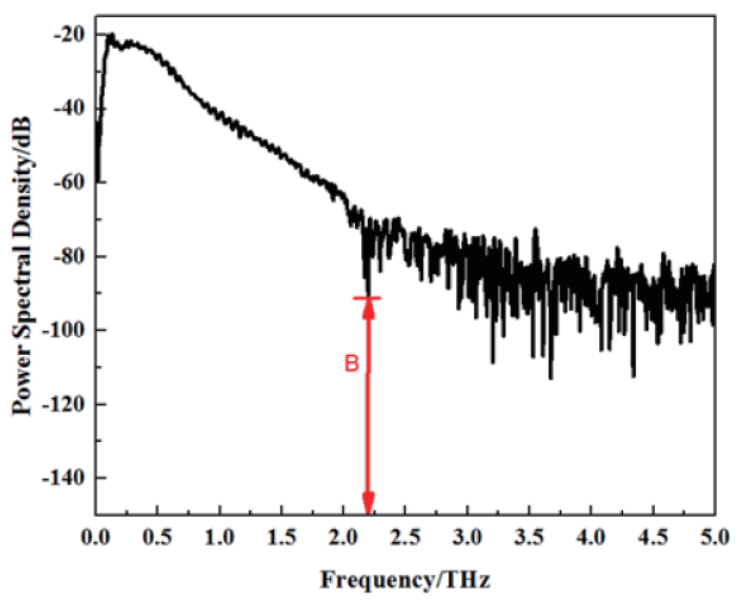

Fig. 3. (Color online) Information of terahertz imaging in the frequency domain.

$$
E=\int_{-\infty}^{\infty} s^{2}(t) d t=\int_{-\infty}^{\infty}|S(f)|^{2} d f
$$

where $E$ is the energy and $|S(f)|^{2}$ is the spectral energy density.

\subsubsection{Image selection with multi-evaluation index}

The different needs of nondestructive testing require the recognition of various targets in terahertz images to evaluate image quality. Four indexes are used in this study to evaluate the quality of a digital image: ${ }^{(12,13)}$ standard deviation (SD), information entropy (H), average gradient (AG), and spatial frequency (SF). The cutoff frequency of the terahertz transmission spectrum of packaging materials is also used for evaluation. Since H and AG are essential information on the internal defects of an image, they are used as crucial indicators with large weights. The cutoff frequency of the terahertz transmission spectrum of packaging materials is an indicator of noise. The noise is the most important indicator when reconstructing the internal image from the frequency information of an IC chip. After the evaluation indicators of all terahertz images in the image data set are compared and calculated, an image is extracted from the image library to obtain a high-quality terahertz image. The weights of the indexes decrease in the order of terahertz spectrum cutoff frequency $>A G>H>S F>S D$.

\subsection{Image matching based on SIFT feature- $K$-means clustering}

The SIFT is an operator based on invariant technology used to describe a local feature of an image. The SIFT algorithm finds extreme points in spaces of different scales and calculates the size, direction, and scale of the extreme points. The extreme points of information can describe the characteristics of the image. The SIFT features are extracted and matched in the process of comparing the extreme points. A common matching method is to calculate the Euclidean 
distance between SIFT features in two images. ${ }^{(14,15)}$ Since each image generates thousands of SIFT feature points, the matching requires a large amount of calculation, especially when the amount of image data is large.

To improve the efficiency of extraction and matching of the images, the traditional matching method using SIFT feature points is improved in this study. First, the SIFT feature points of all images are extracted, and the $K$-means clustering algorithm is used to cluster these points to generate a new vector with a $K$-dimensional feature for each image. Then, the vectors of different images are matched to each other by a similarity measurement calculation of the two images. Here, the $K$-means clustering algorithm is utilized to perform statistical analysis on SIFT feature points. In this method, only two $K$-dimensional feature vectors of two images are measured, which greatly reduces the computation. This process can also decrease the complexity and improve the performance of the algorithm. The procedure of the $K$-means clustering algorithm for SIFT feature points is as follows.

(1) The SIFT feature points of all images are extracted from the image data set, and a subset $X=$ $\left(X_{1 d}, X_{2 d}, \ldots, X_{n d}\right)$ of the SIFT feature points is selected, where $X_{1 d}(i=1,2, \ldots, n)$ represents the ith feature point. Its dimension $d$ is 128 , that is, each feature point contains a 128-dimensional vector.

(2) $K$ appropriate clusters and $K$ SIFT points are randomly selected from the subset as initial cluster centers (centroids).

(3) The distance from each SIFT point to the cluster center is calculated as the Euclidean distance. Using the Euclidean distance, each SIFT point in the subset is assigned to the nearest centroid in turn to form $K$ clusters.

(4) The cluster centers are iteratively updated by considering the Euclidean distances and using the sum of the squared error (SSE) as the objective function of clustering,

$$
S S E=\sum_{i=1}^{k} \sum_{x \in c_{i}} \operatorname{dist}\left(c_{i}, x\right)^{2},
$$

where $k$ is the number of cluster centers, $c_{i}$ is the number of centers, and dist is the Euclidean distance.

The $K$ th centroid $C_{k}$ is calculated to minimize the SSE as follows.

$$
\begin{gathered}
\frac{\partial}{\partial c_{k}} S S E=\frac{\partial}{\partial c_{k}} \sum_{i=1}^{k} \sum_{x \in c_{i}}\left(c_{i}-x\right)^{2} \\
=\sum_{i=1}^{k} \sum_{x \in c_{i}} \frac{\partial}{\partial c_{k}}\left(c_{i}-x\right)^{2} \Rightarrow \\
=\sum_{x \in c_{k}} 2\left(c_{k}-x_{k}\right)=0 \\
m_{k} c_{k}=\sum_{x \in c_{k}} x_{k} \Rightarrow c_{k}=\frac{1}{m_{k}} \sum_{x \in c_{k}} x_{k}
\end{gathered}
$$


Here, $m_{k}$ is the number of feature points in the $K$ th centroid.

The average of all the feature points in each cluster is calculated using Eq. (2). The average is used to generate the corresponding average vector as the cluster center for calculating the objective function, and the $K$-means test is run twice to produce two different clusters. Then, the average vector with the smallest objective function is regarded as the new centroid.

(5) The convergence of the algorithm is determined to establish whether the clustering center and the objective function have changed. If they are unchanged, the clustering results of $K$ clusters are output. If they have changed, the procedure returns to the third step. The common convergence condition of the algorithm is as follows: when the cluster does not change, that is, no new feature points are redistributed, the maximum number of iterations is reached. Here, the cluster no longer changes according to the algorithm convergence condition.

(6) The cosine similarity is used to distinguish various images. First, the cosine value of the angle between the feature vectors of two images is calculated. All image feature vectors in the image data set are sorted according to the cosine values, and eight images with the largest cosine values are selected as the best matched images.

\subsection{Image fusion based on wavelet transform}

The wavelet transform is typically used in frequency-domain image fusion. ${ }^{(16)}$ The wavelet transform can deal with the low-frequency information of the image and high-frequency information in horizontal, vertical, and diagonal directions at the same time. By choosing a suitable wavelet basis and a suitable number of decomposition layers, the noise is reduced and edges are sharpened. Image fusion based on the wavelet transform transforms the fusion coefficients of multiple images inversely to obtain the final fused image. To enhance image fusion efficiency, the data of multiple images to be fused in different frequency bands are processed by a wavelet-transform-based fusion method. A flowchart of the image fusion algorithm based on the wavelet transform is shown in Fig. 4.

\section{Results and Discussion}

\subsection{Terahertz spectral scan in time domain}

Three failed IC chips, in which faults were generated by applying a high voltage, were selected in the experiment: a ceramic SDIP40 packaged chip (Sample 1), an epoxy resin SDIP40 packaged chip (Sample 2), and an epoxy resin LCC21 packaged chip (Sample 3). During the experiment, a terahertz wave transmitter and a terahertz receiver were fixed on a horizontal optical rail. The samples were placed on a $2 \mathrm{D}$ scanning platform that moved in the $\mathrm{x}$-and $\mathrm{y}$-axis directions from the focus position of the terahertz pulse. The terahertz spectra of the samples were acquired by $2 \mathrm{D}$ scanning as shown in Fig. 5 .

Figure 5(b) shows that the terahertz pulse effectively penetrates the three different samples. The time-domain spectra of the samples appear behind the reference spectrum on the timeline, and the amplitude of the electric field intensity after transmission is attenuated to some extent. 


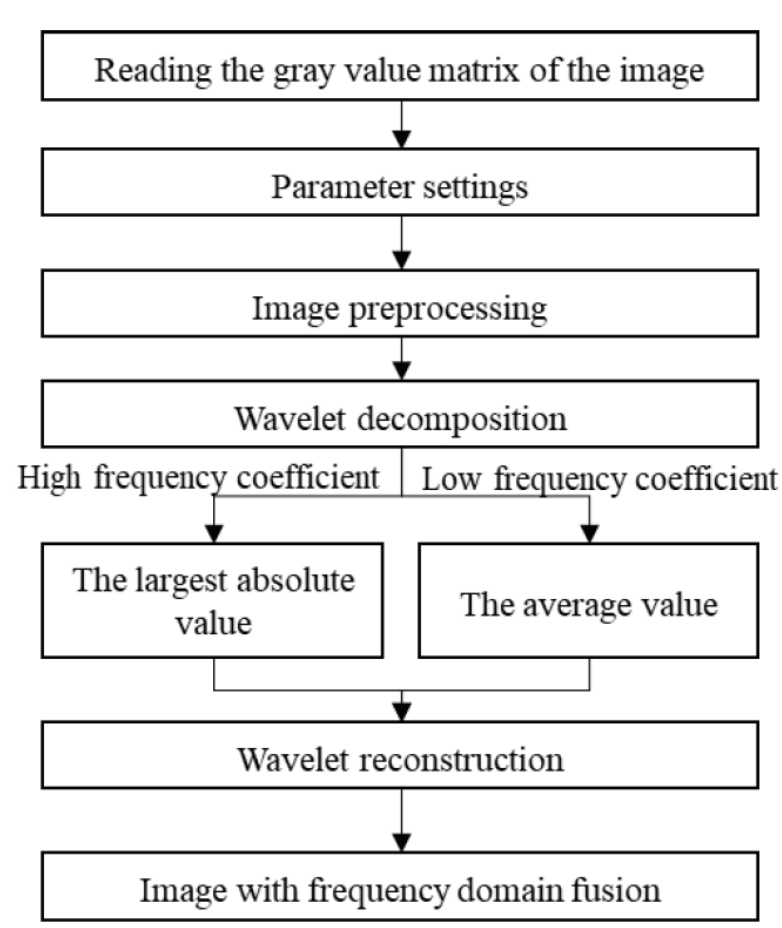

Fig. 4. Flowchart of image fusion algorithm based on wavelet transform.

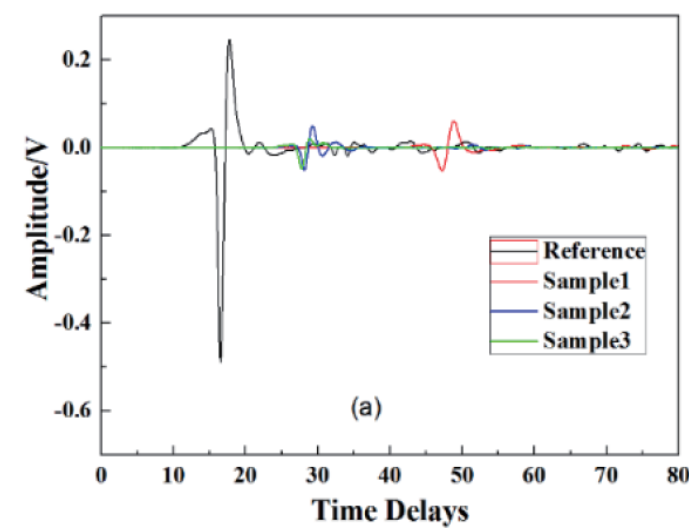

(a)

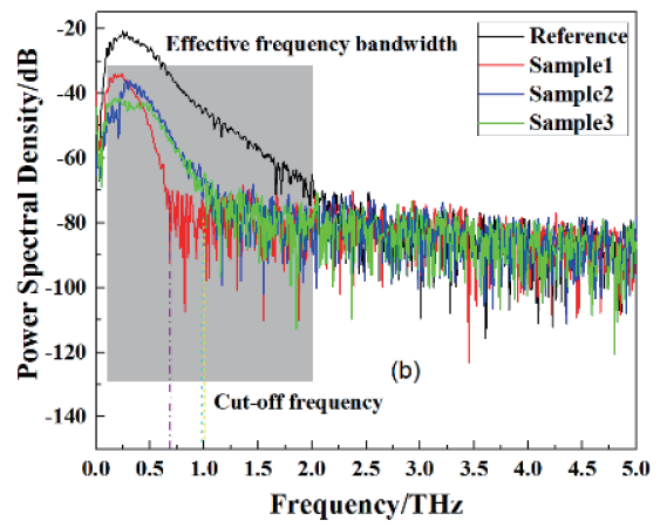

(b)

Fig. 5. (Color online) (a) Transmission spectra of samples in the time domain; (b) transmission spectra of samples in the frequency domain.

Although Sample 3 (green line) shows a larger attenuation than Samples 1 (red line) and 2 (blue line), their peak values are almost the same. The transmission spectrum in the frequency domain [Fig. 5(b)] reveals that the effective frequency bandwidth for terahertz imaging is about 0.2-2.0 $\mathrm{THz}$ (gray area in the figure). The cutoff frequencies of Samples 1-3 are about 0.7, 1.0, and 1.0 $\mathrm{THz}$, respectively. The difference in the frequency originates from the different packaging materials. 


\subsection{Multimodal imaging and image quality evaluation}

A multimodal imaging process is used to generate a spectral image data set of samples. Six images are drawn from the time domain to obtain information on the electric field intensity. Each image contains the maximum peak amplitude, minimum peak amplitude, peak-to-peak amplitude, average amplitude, maximum peak phase of the amplitude, and minimum peak phase of the amplitude. The number of images used in energy information imaging with a frequency interval of $3.125 \mathrm{GHz}$ is 1120 in the frequency domain. The total number of terahertz images of each sample is 1126. Multiple evaluation indicators are used to evaluate the quality of images in the data set. Figures 6-8 show the quantitative experimental results of different imaging modes.

The experimental results show that the terahertz image quality is poor for the indirect imaging of the peak electric field intensity and phase information in the time domain. The effect of the energy-based imaging at different frequencies in the frequency domain is better than that of imaging using time-domain information (Figs. 6-8).

A comparison of the imaging effects of terahertz transmission energy at different frequencies reveals that (1) the terahertz image resolution of the three samples at a low frequency is low owing to the low wavelength of the terahertz wave and is affected by the diffraction limit, (2) the internal defects of the sample are clearly revealed in the mid-frequency range, and (3) the energy

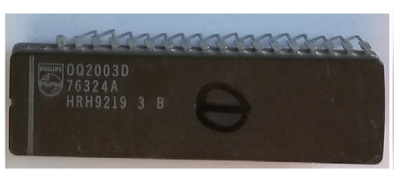

(a) Optical image

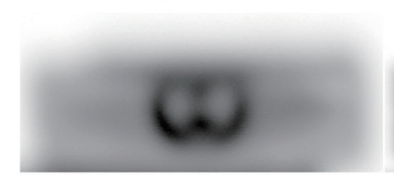

(e) Energy imaging at $0.25937 \mathrm{THz}$

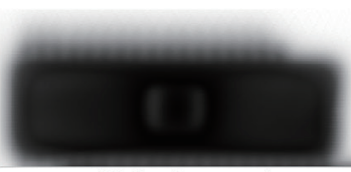

(b) Peak-to-peak amplitude imaging

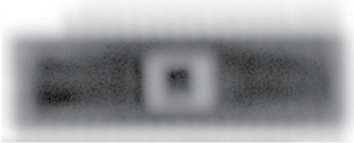

(f) Energy imaging at $0.575 \mathrm{THz}$

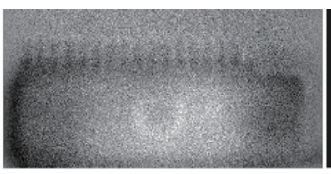

(c) Average amplitude imaging

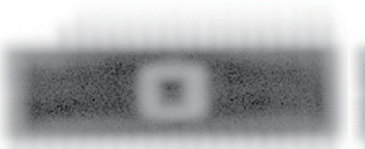

(g) Energy imaging at $0.6375 \mathrm{THz}$

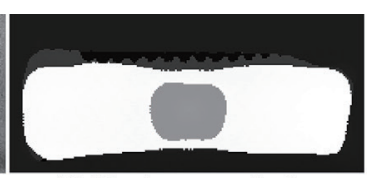

(d) Maximum peak phase amplitude imaging

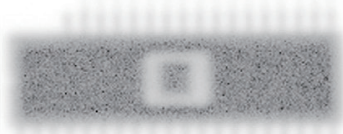

(h) Energy imaging at $0.90937 \mathrm{THz}$

Fig. 6. (Color online) Comparison of imaging results of ceramic SDIP40 packaged chip (Sample 1).

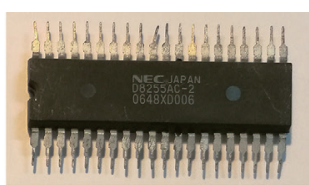

(a) Optical image

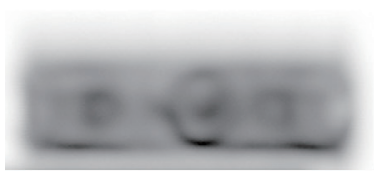

(e) Energy imaging at $0.36875 \mathrm{THz}$

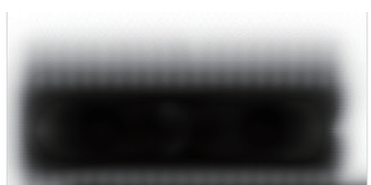

(b) Peak-to-peak amplitude imaging

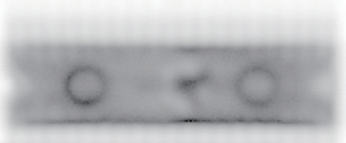

(f) Energy imaging at $0.61875 \mathrm{THz}$

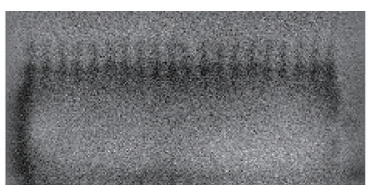

(c) Average amplitude imaging

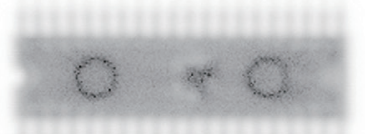

(g) Energy imaging at $0.83125 \mathrm{THz}$

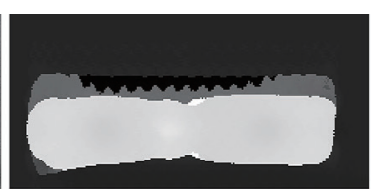

(d) Maximum peak phase amplitude imaging

(h) Energy imaging at $1.0781 \mathrm{THz}$

Fig. 7. (Color online) Comparison of imaging results of epoxy resin SDIP40 packaged chip (Sample 2). 


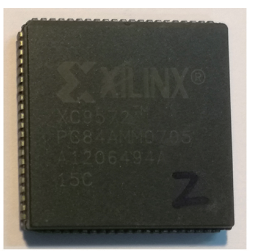

(a) Optical image

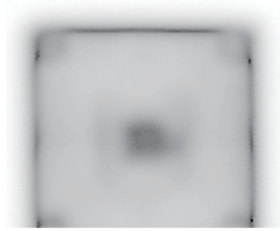

(e) Energy imaging at $0.5 \mathrm{THz}$

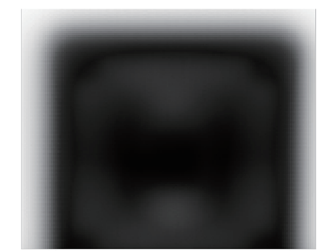

(b) Peak-to-peak amplitude imaging

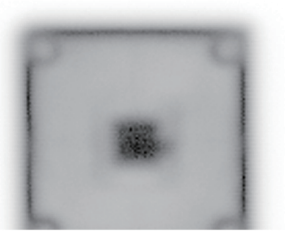

(f) Energy imaging at $0.66562 \mathrm{THz}$

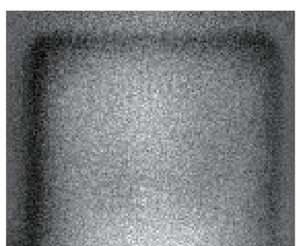

(c) Average amplitude imaging

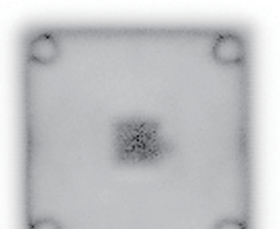

(g) Energy imaging at $0.7875 \mathrm{THz}$

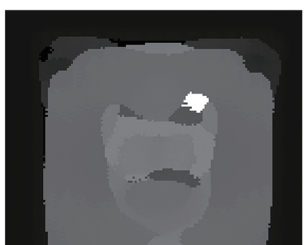

(d) Maximum peak phase amplitude imaging

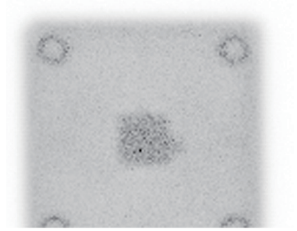

(h) Energy imaging at $0.9875 \mathrm{THz}$

Fig. 8. (Color online) Comparison of imaging results of epoxy resin LCC21 packaged chip (Sample 3).

of the terahertz wave in a high-frequency band gradually decreases, which results in its reduced penetration.

Therefore, although the terahertz images have a higher resolution in the high-frequency band than in the low-frequency band, the image noise increases. As the frequency increases, the useful information of the image is gradually masked, making it impossible to effectively reveal the inside of the samples. Therefore, the image quality evaluation indicators are necessary to evaluate the images acquired from the library and improve the images. The evaluation results are shown in Table 1.

According to the objective evaluation indexes in Table 1, Sample 1 has the best comprehensive evaluation indicators for energy imaging at $0.6375 \mathrm{THz}$. The outline and internal edges of the image are clear in Fig. 6(g). The internal die area has sharp edges, which implies that the terahertz image is a representative image of Sample 1. The packaging materials of Samples 2 and 3 are the same, so the terahertz transmission spectra are similar [Fig. 5(b)] and the evaluation indicators are similar. Samples 2 and 3 have the best evaluation results of energy imaging at 0.83125 and $0.66562 \mathrm{THz}$, respectively. Compared with other images, the values of AG, H, and $\mathrm{SF}$ at the selected representative image are higher. For instance, for Sample 2, the values of AG and SF of the images at $0.83125 \mathrm{THz}$ are higher than those of the images at 0.36875 and 0.61875 $\mathrm{THz}$, but the value of $\mathrm{H}$ is slightly smaller. Although the values of AG and SF are higher for the images at $1.0781 \mathrm{THz}$, these images are noisy, as shown in Fig. 7(h). Figures 7(g) and 8(f) show the positioning hole of the packaging and an internal die defect, respectively. In particular, Fig. $7(\mathrm{~g})$ reveals pin lead information in the die area, which is not shown in the images of Sample 1. Thus, the best imaging effect for Sample 2 is at $0.66562 \mathrm{THz}$. For Sample 3, the values of AG, $\mathrm{SF}$, and $\mathrm{H}$ for the image at $0.66562 \mathrm{THz}$ are all higher than those for the images at 0.5 and $0.76875 \mathrm{THz}$. Similarly, although the values of AG and SF are higher for the images at 0.9875 $\mathrm{THz}$, these images are noisy, as shown in Fig. 8(h). Therefore, these two images (imaging at $0.83125 \mathrm{THz}$ for Sample 2 and $0.66562 \mathrm{THz}$ for Sample 3) are regarded as representative terahertz images of the samples. 
Table 1

Comparison of objective evaluation indexes for image quality.

\begin{tabular}{lcccc}
\hline Sample 1 & SD & H & AG & \multicolumn{1}{c}{ SF } \\
\hline Peak-to-peak amplitude imaging & 96.4144 & 7.1958 & 1.5208 & 8.4430 \\
Maximum peak phase of amplitude imaging & 104.7158 & 3.1689 & 2.5543 & 25.2883 \\
Energy imaging at $0.25937 \mathrm{THz}$ & 68.6096 & 7.3271 & 2.1801 & 3.8016 \\
Energy imaging at $0.575 \mathrm{THz}$ & 81.2686 & 6.7650 & 3.6218 & 6.9800 \\
Energy imaging at $0.6375 \mathrm{THz}$ & 77.8948 & 6.6411 & 4.6425 & 9.4534 \\
Energy imaging at $0.90937 \mathrm{THz}$ & 57.4804 & 6.3358 & 8.1409 & 20.1919 \\
\hline Sample 2 & $\mathrm{SD}$ & $\mathrm{H}$ & $\mathrm{AG}$ & \multicolumn{1}{c}{$\mathrm{SF}$} \\
\hline Peak-to-peak amplitude imaging & 94.2230 & 7.4626 & 3.5304 & 8.7114 \\
Maximum peak phase of amplitude imaging & 84.3356 & 4.6213 & 6.3993 & 26.8121 \\
Energy imaging at $0.36875 \mathrm{THz}$ & 63.8748 & 7.0790 & 2.4595 & 4.9203 \\
Energy imaging at $0.61875 \mathrm{THz}$ & 55.9518 & 6.4963 & 2.8094 & 6.2760 \\
Energy imaging at $0.83125 \mathrm{THz}$ & 49.9157 & 6.3159 & 4.1725 & 10.2933 \\
Energy imaging at $1.0781 \mathrm{THz}$ & 53.4474 & 6.4112 & 8.7161 & 20.8962 \\
\hline Sample 3 & $\mathrm{SD}$ & $\mathrm{H}$ & $\mathrm{AG}$ & $\mathrm{SF}$ \\
\hline Peak-to-peak amplitude imaging & 88.0291 & 7.1496 & 1.2031 & 8.0731 \\
Maximum peak phase of amplitude imaging & 44.4462 & 4.2407 & 1.4056 & 15.9233 \\
Energy imaging at $0.5 \mathrm{THz}$ & 40.1138 & 6.2996 & 2.9991 & 7.9334 \\
Energy imaging at $0.66562 \mathrm{THz}$ & 54.5763 & 6.4473 & 4.1964 & 10.1841 \\
Energy imaging at $0.76875 \mathrm{THz}$ & 44.0045 & 6.0888 & 3.6876 & 8.9417 \\
Energy imaging at $0.9875 \mathrm{THz}$ & 35.9417 & 5.7666 & 5.7284 & 12.6513 \\
\hline
\end{tabular}

\subsection{Representative image matching extraction based on SIFT feature clustering}

The SIFT feature- $K$-means model extracts 1126 terahertz images of each sample as representative images selected by the comprehensive evaluation. The eight images with the highest similarity are selected as the source images for Samples 1-3 as shown in Figs. 9-11, respectively. In the experiment, the Gaussian scale of SIFT feature point extraction is set as 1.6 and the number of $K$-means clusters is $K=500$. Specific model parameter settings are shown in Table 2. The image clustering and matching in this study effectively retrieve the representative images of high quality from a large amount of image data. Clear images are obtained by energy imaging in the frequency domain. Although there is a slight difference in contrast between the images, the details of the images are well revealed, such as the sample outlines and internal defects.

\subsection{Image reconstruction by wavelet fusion}

The quantitative analysis of the SIFT feature $-K$-means model allows eight representative images of high quality of each sample to be used to identify the faults of the samples. To verify the effect of image processing after wavelet fusion, we perform wavelet fusion processing on the representative images of the samples. The image quality evaluation indicators are adopted to compare the effects of each processing. The result of the fusion is shown in Fig. 12, and the objective evaluation results are presented in Table 3. The results indicate that the fusion results and objective evaluation indicators are improved by wavelet fusion. After wavelet fusion, the 


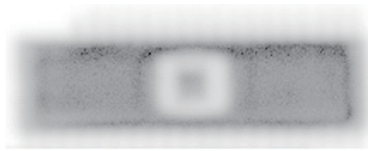

(a) $0.62812 \mathrm{THz}$

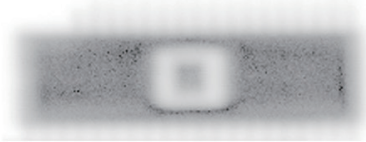

(e) $0.66562 \mathrm{THz}$

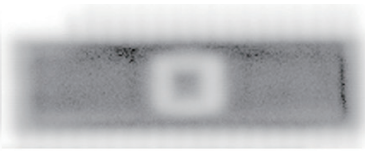

(b) $0.63125 \mathrm{THz}$

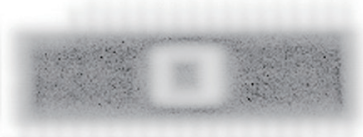

(f) $0.72812 \mathrm{THz}$

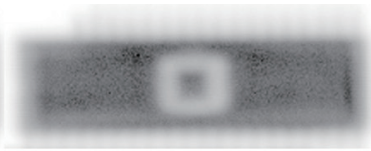

(c) $0.63437 \mathrm{THz}$

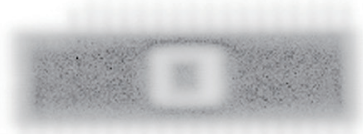

(g) $0.73125 \mathrm{THz}$

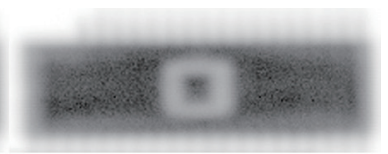

(d) $0.6375 \mathrm{THz}$

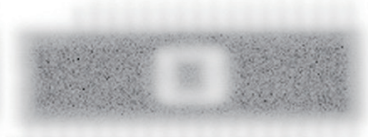

(h) $0.75132 \mathrm{THz}$

Fig. 9. Representative images of Sample 1 at different frequencies.

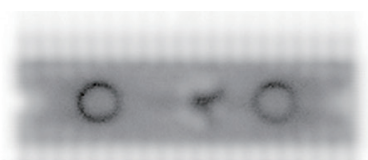

(a) $0.66875 \mathrm{THz}$

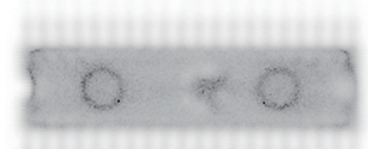

(e) $0.82187 \mathrm{THz}$

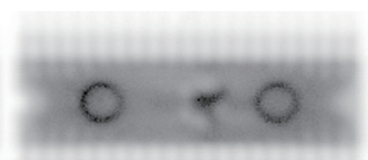

(b) $0.68437 \mathrm{THz}$

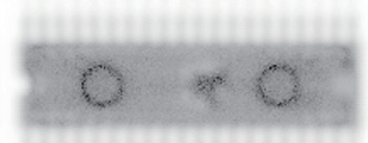

(f) $0.825 \mathrm{THz}$

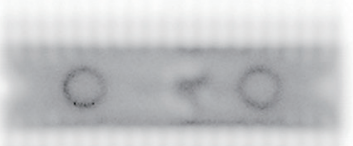

(c) $0.7 \mathrm{THz}$

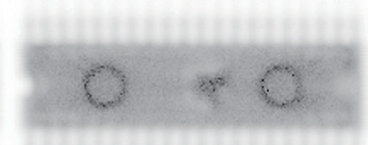

(g) $0.82812 \mathrm{THz}$

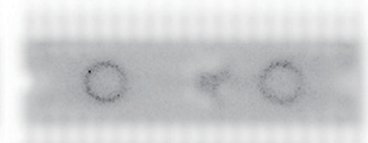

(d) $0.74687 \mathrm{THz}$

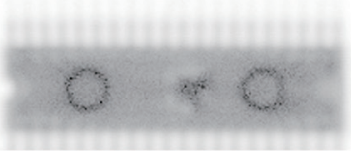

(h) $0.83125 \mathrm{THz}$

Fig. 10. Representative images of Sample 2 at different frequencies.

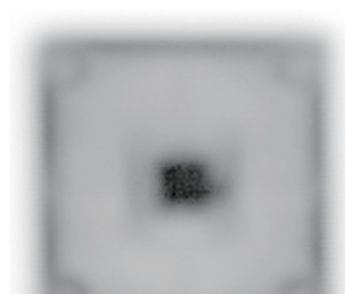

(a) $0.56562 \mathrm{THz}$

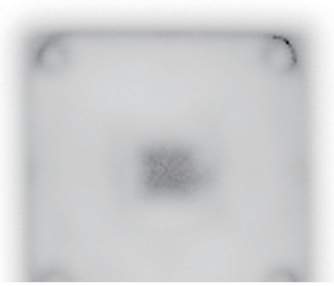

(e) $0.6 \mathrm{THz}$

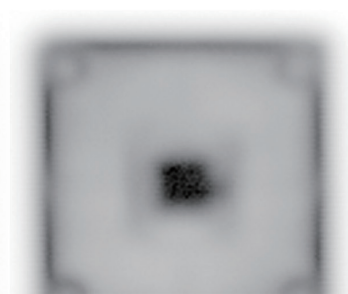

(b) $0.57187 \mathrm{THz}$

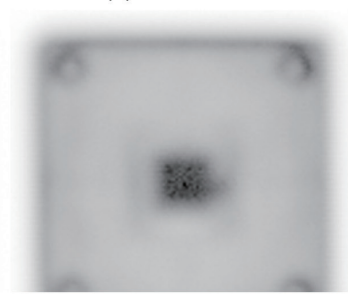

(f) $0.60625 \mathrm{THz}$

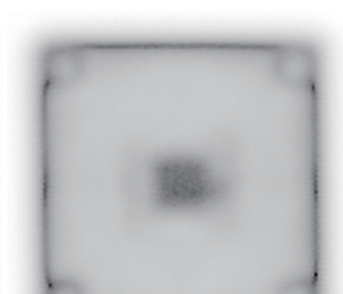

(c) $0.58437 \mathrm{THz}$

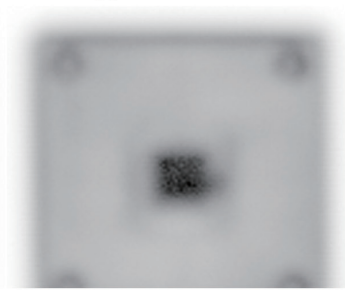

(g) $0.61562 \mathrm{THz}$

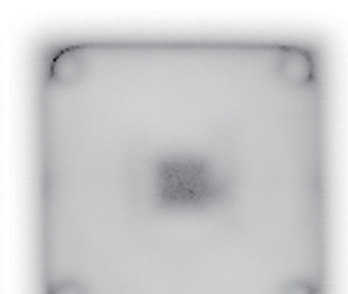

(d) $0.59375 \mathrm{THz}$

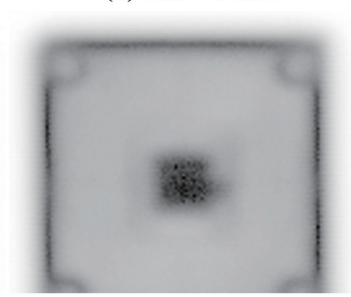

(h) $0.66562 \mathrm{THz}$

Fig. 11. Representative images of Sample 3 at different frequencies.

Table 2

Model parameters of the samples.

\begin{tabular}{lccccccc}
\hline Parameter & $\sigma$ & $S$ & SIFT descriptor & $K$ & Image size & Number of images & Output images \\
\hline Value & 1.6 & 3 & 128 & 500 & $560 \times 368$ & 1126 & 8 \\
\hline
\end{tabular}

image brightness, definition, and contrast are enhanced, while the image information is retained. This demonstrates the effectiveness of wavelet fusion. 


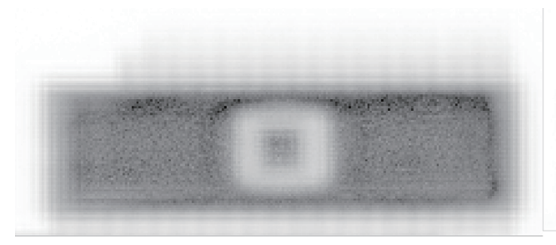

(a) Wavelet image fusion for sample 1

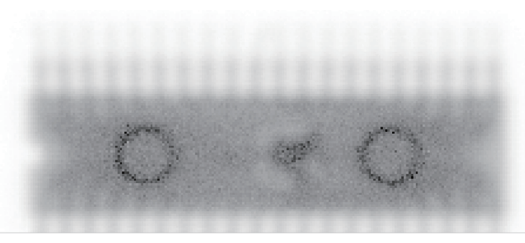

(b) Wavelet image fusion for sample 2

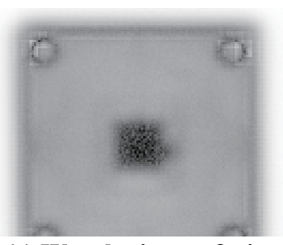

(c) Wavelet image fusion for sample 3

Fig. 12. Results of fusion imaging of samples.

Table 3

Objective evaluation indexes after image fusion.

\begin{tabular}{lcccr}
\hline Sample & SD & H & AG & \multicolumn{1}{c}{ SF } \\
\hline Sample 1 & 28.4969 & 6.3310 & 4.1178 & 11.7488 \\
Sample 2 & 17.1010 & 5.6900 & 4.6641 & 8.2189 \\
Sample 3 & 28.5642 & 6.3324 & 4.1544 & 12.0321 \\
\hline
\end{tabular}

\section{Conclusions}

Terahertz spectral imaging has attracted increasing attention in the field of chip nondestructive testing and analysis. However, in practice, the quality of terahertz spectral images of chips often fails to meet the requirements. To solve this problem, we propose a terahertz image processing method, where a multimode imaging method and image quantitative analysis are used to generate a large number of source images. Moreover, SIFT feature extraction and $K$-means clustering are combined to select high-quality terahertz images. The wavelet fusion image processing method is also used to improve the quality of the terahertz images. Through experimental analysis, it is found that the proposed method can quickly and effectively select high-quality terahertz images from a large number of multiple-source terahertz image data. Moreover, the image clarity, information entropy, and other key indicators are greatly improved compared with those for methods involving processing single terahertz images, enabling the proposed method to meet the requirements of chip failure analysis.

\section{Acknowledgments}

This work was supported by the Natural Science Foundation of Fujian Province, China (Grant Nos. 2020J01714 and 2020J01713).

\section{References}

1 P. Aryan, S. Sampath, and H. Sohn: Sensors 18 (2018) 1981. https://doi.org/10.3390/s18071981

2 G. Li, H. Lee, A. Chattopadhyay. R. Kumar Neerukatti, and K. C. Liu: J. Electron. Packag. 143 (2021) 011002. https://doi.org/10.1115/1.4047228

3 P. Hillger, J. Grzyb, and R. Jain: IEEE Trans. Terahertz Sci. Technol. 9 (2019) 1. https://doi.org/10.1109/ TTHZ.2018.2884852

4 S. H. Park, J. W. Jang, and H. S. Kim: J. Micromech. Microeng. 25 (2015) 095007. https://doi.org/10.1088/09601317/25/9/095007 
5 K. Ahi, S. Shahbazmohamadi, and N. Asadizanjani: Opt. Lasers Eng. 104 (2018) 274. https://doi.org/10.1016/j. optlaseng.2017.07.007

6 Z.R. Zhang, Y. Lu, and C.X. Lv: Opt. Lasers Eng. 138 (2021) 106413. https://doi.org/10.1016/j. optlaseng.2020.106413

7 H. Y. Lee, H. S. Kim, and H. S. Lee: Opt. Eng. 43 (2006) 1. https://doi.org/10.1117/1.2181887

8 T. Kanungo, D. M. Mount, and N. S. Netanyahu: IEEE Trans. Pattern Anal. Mach. Intell. 24 (2002) 881. https:// doi.org/10.1109/TPAMI.2002.1017616

9 Q. Mao, Y. L. Zhu, and C. Lv: Opt. Express 28 (2020) 25293. https://doi.org/10.1364/OE.396604

10 Q. Mao, Y. L. Zhu, and C. Lv: Opt. Express 28 (2020) 5000. https://doi.org/10.1364/OE.384146

11 A. Kiarash and A.Mehdi: Proc. 2016 Terahertz Physics, Devices, and Systems X - Advanced Applications in Industry and Defense (SPIE, 2016) 17. https://doi.org/10.1117/12.2228680

12 Z. Yu, H. R. Wu, and S. Winkler: Proc. 2002 IEEE (IEEE, 2002) 154. https://doi.org/10.1109/5.982412

13 N. B. Nill and B. H. Bouzas: IEEE Signal Process. Lett. 9 (2002) 388. https://doi.org/10.1117/12.56114

14 Y.X. Ye, J. Shan, and L. Bruzzone: IEEE Trans. Geosci. Remote Sens. 55 (2017) 2941. https://doi.org/10.1109/ TGRS.2017.2656380

15 Y. Liu, S. P. Liu, and Z. F. Wang: Inf. Fusion 23 (2015) 139. https://doi.org/10.1016/j.inffus.2014.05.004

16 Q. Wang, X. Li, T. Chang, J. Zhang, L. Liu, and H. Zhou: Infrared Phys. Technol. 97(2019) 326. https://doi. org/10.1016/j.infrared.2019.01.013

\section{About the Authors}

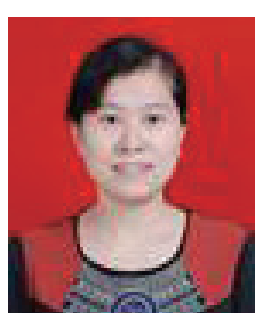

Xiao-Xiang Pu has been an associate professor at Chongqing Electric Power College, China, since 2010. She received her M.S. degree from Chongqing University, China, in 2004. She has published 12 academic articles and three textbooks. Her research interests are in control engineering, electric circuit theory, and electronic technology. (puxiaoxiang_cq@sina.com)

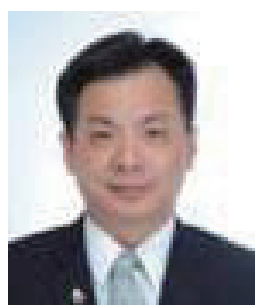

Chih-Cheng Chen has been a professor at Jimei University, China, since 2017. He became a member of IEEE in 2011 and a senior member in 2016. He earned his M.S. degree in 2005 and has recently earned his Ph.D. degree from the Department of Mechatronics Engineering, National Changhua University of Education. He has published 43 academic articles and owns three patents. He has also implemented several projects funded by the National Natural Science Foundation of China, Fujian Province. His research interests include AIoT technology, machine learning, information security, and RFID applications. (chenccheng@fcu.edu.tw).

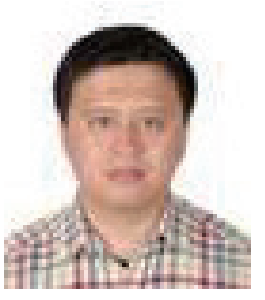

Tie-Jun Li has been an associate professor at Jimei University, China, since 2015. He received his B.S., M.S., and Ph.D. degrees from Chongqing University, China, in 2000, 2005, and 2018, respectively. He has published 21 academic articles and owns 11 patents. He also implemented several projects by the Natural Science Foundation of China, Fujian Province. His research interests are in machine learning, terahertz technology, and sensors. (litiejun@jmu.edu.cn) 


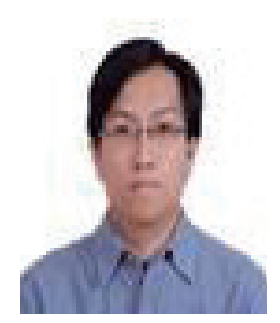

Wei-Lung Mao was born in Taiwan, R.O.C., in 1972. He received his B.S. degree in electrical engineering from National Taiwan University of Science and Technology in 1994 and his M.S. and Ph.D. degrees in electrical engineering from National Taiwan University in 1996 and 2004, respectively. $\mathrm{He}$ is now a professor in the Department of Electrical Engineering and Graduate School of Engineering Science and Technology, National Yunlin University of Science and Technology. His research interests are in satellite navigation systems, intelligent and adaptive control systems, adaptive signal processing, neural networks, and precision control. (wlmao@yuntech.edu.tw)

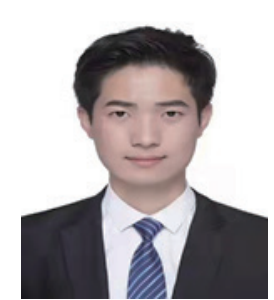

Zhen Liu is currently studying for a master's degree at Jimei University, China. His research interests include deep learning, signal processing, image recognition, and bearing fault diagnosis. 